\title{
Factors affecting the future employment of new graduate nurses as home-visiting nurses: a cross-sectional study in Japan
}

\author{
Yumi Fukuyama ${ }^{1}$, Akina Ishibashi ${ }^{1}, K$ Koichi Shinchi ${ }^{1}$, and Akiko Akiyama ${ }^{2}$ \\ ${ }^{1}$ Institute of Nursing, Faculty of Medicine, Saga University, Japan \\ ${ }^{2}$ Institute of Nursing and Medical Care, Faculty of Health Science, Kio University, Japan
}

\begin{abstract}
Objective: In Japan, home-visiting nurse (HVN) stations are at the frontline of providing home-based medical care and end-oflife care. The nursing authorities aim to establish an education program that allows new graduate nurses to become home-visiting nurses. However, previous studies have indicated gaps in education between new graduates recently employed as HVNs and experienced home-visiting nurses. The present study further investigates the factors influencing the recruitment of new graduates as home-visiting nurses.

Methods: Self-administered questionnaires were sent to 2,000 HVN stations randomly selected from the 5,565 registered homevisiting nurse stations throughout Japan. The survey covered three main areas, namely, those concerning the respondent (6 items), the home-visiting nurse station (8 items), and the nursing services provided (12 items).

Results: Four of the 26 items were statistically significant, and only one of these was determined by multivariate logistic regression analysis to be an independent factor for accepting new graduates as home-visiting nurses. This factor was undergraduate homevisiting nurse training for student nurses $(\mathrm{OR}=1.916, \mathrm{CI}=1.124-3.267)$.

Conclusion: To increase the recruitment of new graduates as home-visiting nurses, these findings suggest that nursing schools nationwide and home-visiting nurse stations should further cooperate with the specific aim of increasing the provision of practical training at home-visiting nurse stations for pre-graduation student nurses.
\end{abstract}

Key words: home care, home-visiting nurse, new graduates, Japan

\section{Introduction}

Japan has the highest proportion of elderly people in the world, with a growing number receiving long-term care in their own homes. Increasing the number of clinics and homevisiting nurse (HVN) stations is essential for adequate home medical care. However, despite the recent rise in this number of institutions, increasing the number of nursing staff is now the biggest issue faced by Japan in this area ${ }^{1-3)}$. The

Received: May 19, 2021

Accepted: September 13, 2021

Correspondence: Yumi Fukuyama, Institute of Nursing, Faculty of Medicine, Saga University, 5-1-1 Nabeshima, Saga 849-8501, Japan E-mail: yumifuku@cc.saga-u.ac.jp

This is an open-access article distributed under the terms of the Creative Commons Attribution Non-Commercial No Derivatives
(by-nc-nd) License $<$ http://creativecommons.org/ licenses/by-nc-nd/4.0/>.
Japanese Nursing Association is making efforts to promote nurse retention and support for job transfer/placement assistance (such as the re-employment of nurses who had earlier decided to leave the profession for personal reasons such as child-care or moving residence etc.) to secure more nurses in the field of home medical care ${ }^{4}$. In the past, experienced hospital nurses have transferred to the emerging field of HVN. The average age of HVNs is 47.0 years old ${ }^{5)}$, which is well above the average age of a hospital nurse. There is therefore some concern that the HVN field may shrink if more young graduate nurses are not introduced. To address this, many nursing associations at the local prefectural level are looking at the possibility of employing new graduates directly as HVNs.

Previous studies have suggested that working at an HVN station is not a realistic option for any new graduate because specialized and extensive experience is needed to work as an $\mathrm{HVN}^{6,7)}$. Therefore, we conducted a qualitative study asking skilled HVNs to describe the characteristics 
required to perform their duties effectively. Our study results identified the following two major categories for the qualities required for new graduates to work as HVNs: 1) basic knowledge and skills equivalent those required for the national exam for registered nurses, and 2) the appropriate attitude as members of society ${ }^{8)}$.

We have also previously conducted a qualitative study to explore the actual conditions in practice by identifying the difficulties and challenges reported first-hand directly by new graduates working as HVNs ${ }^{9}$.

Finally, here we report our new findings after investigating the HVNs' opinions and actual conditions at HVN stations to identify and define the factors influencing the acceptance and recruitment of new graduate working as HVNs.

\section{Methods}

\section{Sample selection}

A cross-sectional study was conducted from August to September 2019 in Japan. We used the stratified sampling method to randomly select 2,000 of the 5,565 registered HVN stations nationwide, representing $30.7-43.4 \%$ of the total number of HVN stations in each of the seven regional districts of Japan, as follows: Hokkaido (124/286), Tohoku (118/335), Kanto (578/1,843), Chubu (305/837), Kinki (441/1,437), Chugoku/Shikoku (173/508), and Kyushu/Okinawa (261/733) (Figure 1).

Of the 2,000 HVN stations selected, the records of 74 had incomplete addresses, and the questionnaire survey was consequently sent out by letter post to a total of $1,926 \mathrm{HVN}$ stations. Among the 328 responses received (response rate $17.0 \%), 326$ contained valid answers, which were then subjected to statistical analysis.

This study survey included an introductory cover letter that clearly stated the study purpose, the right to refuse to participate, the strict privacy protection, and the safeguarding of the data, with the exception of publication of statistically analyzed anonymized data. Returning an answered questionnaire was, therefore, considered consent. This survey protocol was approved by the ethics committee of Kio University, Nara, Japan (approval number: H30-21).

This study included an introductory cover letter which clearly stated the study purpose, the right to refuse to participate, the enacted privacy protection and strict safeguarding of the data-except for the publication of statistically analyzed anonymized data. Returning an answered questionnaire was therefore considered to be consent. This survey protocol was approved by the ethics committee at Kio University, Nara, Japan (approval number: H30-21).

\section{Measurements}

As no validated research tools existed to achieve the objectives of our study, the questionnaire was developed based on 26 factors related to the possibility of accepting new graduates as HVNs from previous reports ${ }^{10-12)}$. Subjects were asked to assign a score ranging from 1 (highly disagree) to 5 (highly agree). The questionnaire was piloted and revealed high internal reliability with a Cronbach's alpha of 0.85 .

\section{Statistical analysis}

We conducted item analysis followed by Chi-squared test or t-test, dividing between acceptance (score 4 or 5) and non-acceptance (score 1, 2, or 3) of new graduates in HVN station. The multicollinearity of the 26 items was confirmed by linear regression analysis, and the results are shown in Table 1. Since the variance inflation values (VIF) values of the 26 items ranged from 1.3 to 8.7 , it was determined that there was no multicollinearity, and all 26 items were adopted as independent variables. We then selected items with a $P$-value $<0.05$ as independent variables for multivariate logistic regression. Statistical analysis was performed using SPSS $22.0 \mathrm{~J}$ software for Windows.

\section{Results}

Table 1 presents the basic characteristics of the participants. As shown in Table 1, for the $326 \mathrm{HVN}$ facilities in the final sample, the mean age of the HVN station manager was 51.7 years, and the mean number of nurses in the office was 7.0. The acceptance group consisted of $32.8 \%$ of the participants, while the non-acceptance group consisted of $67.2 \%$.

Table 2 shows the correlation between the possible acceptance of new graduates as HVNs and their desired basic characteristics. There was a statistically significant difference in four of the 26 items in the $\chi^{2}$ test: 1) Attached other facility $(P=0.016)$ : the HVN stations which are attached to some other facility such as a large hospital responded that they would accept new graduates in their own HVN station, more so than those that did not have an attached facility, and 2) Providing practical training for pre-graduation student nurses $(P=0.001)$ : the HVN stations that did provide practical training for pre-graduation student nurses responded they were more likely to accept new graduates in their own HVN station than those that did not host pre-graduate student training, and 3) Accepting medical care in your $H V N$ station - tracheal intubation $(P=0.022)$ : the HVN stations that provided medical care involving the use of tracheal intubation were more likely to accept new graduates than those that did not, and 4) Accepting medical care in your $H V N$ station - home ventilator therapy $(P=0.043)$ : the HVN stations that provided care involving home ventilator therapy were more likely to accept new graduates than those that did not.

Table 3 shows the findings from the multivariate logis- 


\section{Journal of Rural Medicine}

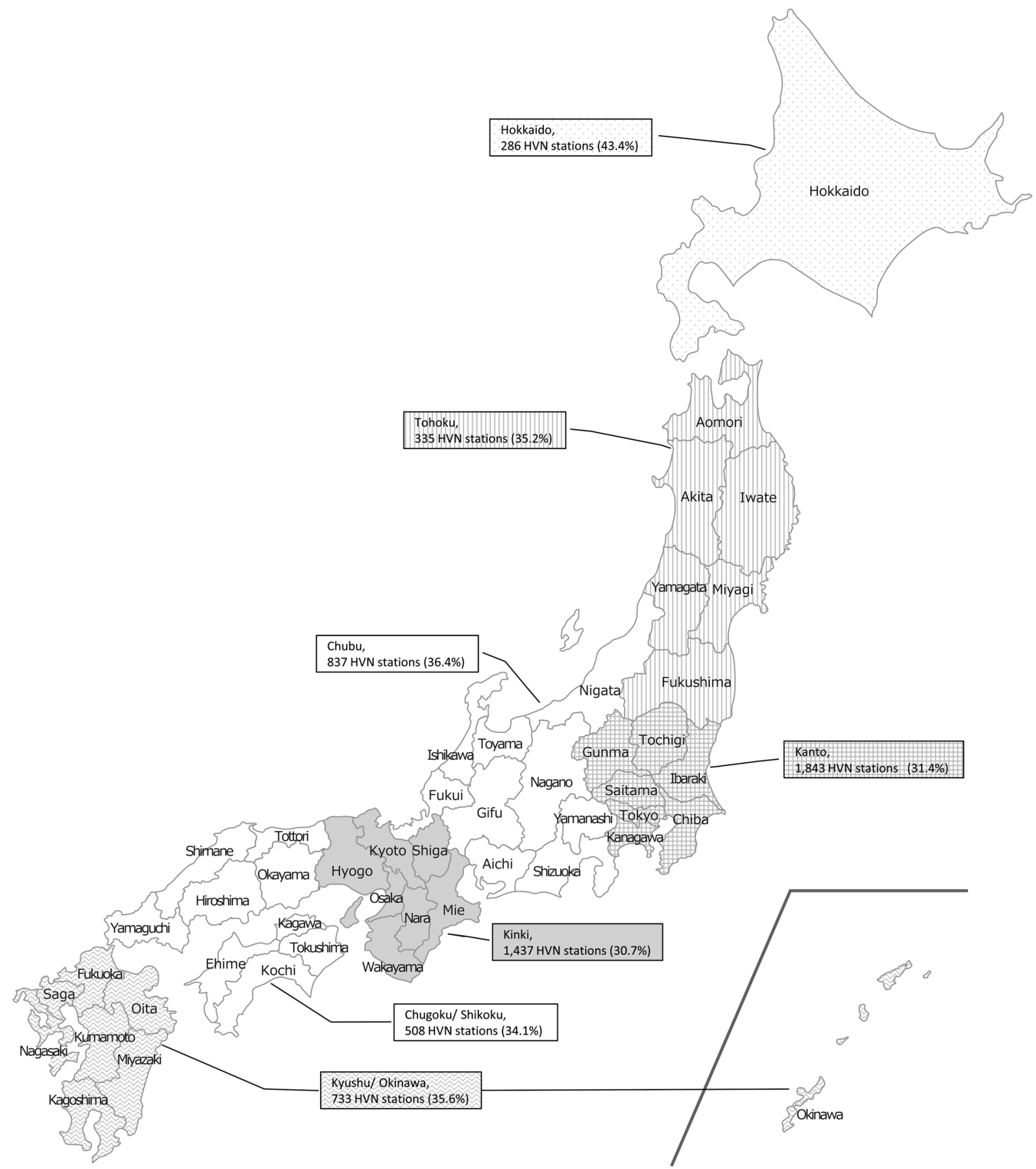

Figure 1 Home-Visiting Nurses (HVN) Stations in Japan and Questionnaire Distribution Rate.

Widely used seven regional divisions of Japan: Hokkaido, Tohoku, Kanto, Chubu, Kinki, Chugoku/Shikoku, and Kyushu/ Okinawa. (): distribution rate.

tic regression analysis. Only one item, Providing practical training for student nurses, was correlated with accepting new graduates to be HVNs (OR=1.916, $\mathrm{CI}=1.124-3.267)$; those HVN stations that provided training to pre-graduation student nurses were 1.9 times more likely to accept new graduates as HVNs than those that did not.

\section{Discussion}

We identified the items influencing the possibility and practicality of accepting new graduates as HVNs. Those HVN stations that provided training to pre-graduation student nurses were almost twice as likely to accept new gradu- 
Table 1. Basic characteristics $(\mathrm{N}=326)$

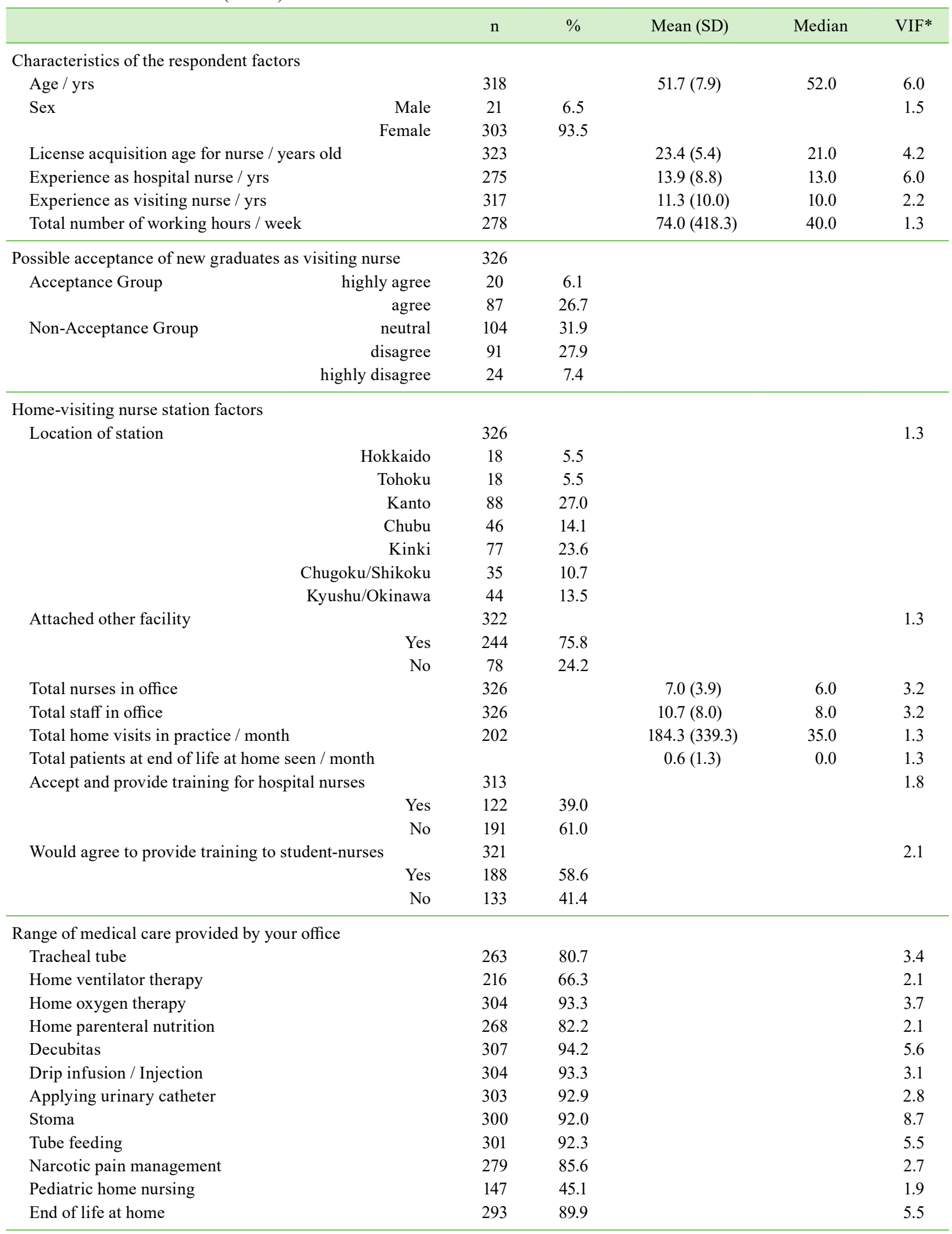

*: Variance Inflation Factor by linear regression analysis. 
Table 2. The relationship between possibility of acceptance of new graduates in home-visiting nurse $(\mathrm{HVN})$ stations and basic characteristics $(\mathrm{N}=326)$

\begin{tabular}{|c|c|c|c|c|c|c|c|}
\hline & & \multicolumn{2}{|c|}{$\begin{array}{l}\text { Acceptance Group } \\
\qquad n=107\end{array}$} & \multicolumn{2}{|c|}{$\begin{array}{l}\text { Non-Acceptance Group } \\
\qquad n=219\end{array}$} & \multirow[t]{2}{*}{$\chi^{2}$} & \multirow[t]{2}{*}{$P$-value } \\
\hline & & $\mathrm{n}$ & Mean (SD) & $\mathrm{n}$ & Mean (SD) & & \\
\hline \multicolumn{8}{|l|}{ Characteristics of the respondent factors } \\
\hline 1. Age / yrs & & & $51.7(8.3)$ & & $51.7(7.7)$ & & 0.965 \\
\hline 2. Sex & Male & 6 & & 15 & & 0.151 & 0.698 \\
\hline 3. License acquisition age for nurse / years old & & & $24.2(5.8)$ & & $23.1(5.2)$ & & 0.102 \\
\hline 4. Experience as hospital nurse / yrs & & & $13.1(8.4)$ & & $14.3(9.0)$ & & 0.330 \\
\hline 5. Experience as visiting nurse / yrs & & & $12.1(7.5)$ & & $11.0(8.1)$ & & 0.214 \\
\hline 6. Total number of working hours / week & & & $45.5(24.7)$ & & $88.4(512.3)$ & & 0.420 \\
\hline \multicolumn{8}{|l|}{ Home-visiting nurse station factors } \\
\hline \multirow[t]{6}{*}{ 7. Location of station } & Hokkaido & 8 & & 10 & & 5.721 & 0.455 \\
\hline & Kanto & 29 & & 59 & & & \\
\hline & Chubu & 11 & & 35 & & & \\
\hline & Kansai & 22 & & 55 & & & \\
\hline & Chugoku/Shikoku & 14 & & 21 & & & \\
\hline & Kyushu/Okinawa & 18 & & 26 & & & \\
\hline \multirow[t]{2}{*}{ 8. Attached other facility } & Yes & 89 & & 155 & & 5.769 & 0.016 \\
\hline & No & 17 & & 61 & & & \\
\hline 9. Total nurses in office & & & $7.6(4.1)$ & & $6.7(3.8)$ & & 0.078 \\
\hline 10. Total staff in office & & & $11.3(7.2)$ & & $10.4(7.9)$ & & 0.320 \\
\hline 11. Total home visits in practice / month & & & $144.4(238.0)$ & & $209.1(388.9)$ & & 0.340 \\
\hline 12. Total patients at end of life at home seen / month & & & $0.71(1.6)$ & & $0.50(1.0)$ & & 0.246 \\
\hline \multirow[t]{2}{*}{ 13. Accept and provide training for hospital nurses } & Yes & 44 & & 78 & & 1.558 & 0.212 \\
\hline & No & 56 & & 135 & & & \\
\hline 14. Would agree to provide training to student-nurses & Yes & 75 & & 113 & & 11.637 & 0.001 \\
\hline \multicolumn{8}{|l|}{ Range of medical care provided by your office } \\
\hline \multirow[t]{2}{*}{ 15. Tracheal tube } & Yes & 94 & & 169 & & 5.260 & 0.022 \\
\hline & No & 13 & & 50 & & & \\
\hline \multirow[t]{2}{*}{ 16. Home ventilator therapy } & Yes & 79 & & 137 & & 4.087 & 0.043 \\
\hline & No & 28 & & 82 & & & \\
\hline \multirow[t]{2}{*}{ 17. Home oxygen therapy } & Yes & 99 & & 205 & & 0.134 & 0.714 \\
\hline & No & 8 & & 14 & & & \\
\hline \multirow[t]{2}{*}{ 18. Home parenteral nutrition } & Yes & 91 & & 177 & & 0.877 & 0.349 \\
\hline & No & 16 & & 42 & & & \\
\hline \multirow[t]{2}{*}{ 19. Decubitas } & Yes & 100 & & 207 & & 0.148 & 0.701 \\
\hline & No & 7 & & 12 & & & \\
\hline \multirow[t]{2}{*}{ 20. Drip infusion / Injection } & Yes & 102 & & 202 & & 0.148 & 0.701 \\
\hline & No & 5 & & 17 & & & \\
\hline \multirow[t]{2}{*}{ 21. Applying urinary catheter } & Yes & 99 & & 204 & & 0.043 & 0.835 \\
\hline & No & 8 & & 15 & & & \\
\hline 22. Stoma & Yes & 97 & & 203 & & 0.408 & 0.523 \\
\hline & No & 10 & & 16 & & & \\
\hline 23. Tube feeding & Yes & 99 & & 202 & & 0.008 & 0.927 \\
\hline & No & 8 & & 17 & & & \\
\hline 24. Narcotic pain management & Yes & 94 & & 185 & & 0.664 & 0.415 \\
\hline & No & 13 & & 34 & & & \\
\hline 25. Pediatric home nursing & Yes & 53 & & 94 & & 1.269 & 0.260 \\
\hline & No & 54 & & 125 & & & \\
\hline 26. End of life at home & Yes & 98 & & 195 & & 1.269 & 0.260 \\
\hline & No & 9 & & 24 & & & \\
\hline
\end{tabular}

$\chi^{2}$ test/t-test. 
Table 3. Acceptance of new graduates as home-visiting nurses (HVNs) and multivariate logistic regression (N=326)

\begin{tabular}{|c|c|c|c|c|}
\hline & & Odds-ratio & $95 \% \mathrm{CI}$ & $P$-value \\
\hline \multirow[t]{2}{*}{ 8. Attached other facility } & Yes & 1.762 & $0.953-3.261$ & 0.071 \\
\hline & No & & & \\
\hline \multirow{2}{*}{ 14. Would provide practical training to student-nurses } & Yes & 1.916 & $1.124-3.267$ & 0.017 \\
\hline & No & & & \\
\hline \multirow[t]{2}{*}{ 15. Tracheal tube } & Yes & 1.344 & $0.565-3.197$ & 0.504 \\
\hline & No & & & \\
\hline \multirow[t]{2}{*}{ 16. Home ventilator therapy } & Yes & 1.270 & $0.638-2.528$ & 0.496 \\
\hline & No & & & \\
\hline
\end{tabular}

Dependent variables: acceptance of new graduates as visiting nurses 0 (Non-Acceptance Group) vs. 1 (Acceptance Group). Independent variables: all four variables 0 (No), 1 (Yes).

ates as HVNs than those that did not.

The National Association for Home-Visiting Nurse Service has reported that new graduates are being hired by (a) 'HVN stations that are attached to some other facility, such as a hospital', and by (b) 'HVN stations already having a large number of HVNs' and depending on the (c) 'type of medical care' offered ${ }^{10)}$. Another study has reported that (d) 'HVN stations which already provided the HVN training for hospital nurses tended to be willing to accept new graduates'11).The present study's findings were consistent with these other reports on one point only, namely that (a) 'the HVN station was attached to some other facility, such as a hospital'. However, our findings are not consistent with those of other reports concerning (b) 'the type of medical care', (c) having 'a large number of HVNs', and (d) 'provided HVN training for hospital nurses'.

At the design stage of our present study, we considered that it might be difficult to accept new graduates into an HVN station that provided medical care involving home ventilator therapy and/or pediatric home nursing. However, our findings indicate the opposite. We believe that the previous report ${ }^{10)}$ was not based on scientific research methods. Another study reported that the characteristics required for new graduate HVNs were 1) relevant basic knowledge, 2) relevant skills, and 3) consideration of the patient and his/ her family members ${ }^{8)}$. The findings of the present study are consistent with those findings. In order to accept new graduates as HVNs, it is necessary to promote cooperation between nursing schools and HVN stations so that pre-graduation student nurses can increase their practical training at HVN stations.

This study found no statistically significant difference between the size of the HVN station and the acceptance of new graduates. However, other reports have suggested that it is difficult to hire new graduates at small HVN stations that do not have in-house training for new employees ${ }^{10)}$, and our previous studies found that new graduate HVNs experienced difficulties in applying what they had learned in nursing school into practice in the home medical area ${ }^{9}$.
The present study's findings suggest a gap between the education received at nursing schools and the real-world challenges which new graduates could face as HVNs. A number of older reports have suggested virtual-reality simulation training $^{13)}$ or high-fidelity simulation ${ }^{14)}$ to help bridge the gap between nursing education and HVN practice.

The limitations of this study are as follows: First, the response rate was fairly low at $17.0 \%$. Second, this study was conducted only on HVNs. Third, this study did not examine the criterion validity. The results of this study may not be generalizable to other settings. It is suggested that future studies should examine a larger number of subjects.

\section{Conclusion}

We have identified the factors influencing the possibility of accepting new graduates as HVNs. The HVN stations that provided training to student nurses were 1.9 times more likely to accept new graduates as HVNs than those that did not.

In conclusion, to increase the number of new graduate nurses wanting to become HVNs and being accepted as HVNs, these findings indicate that it is necessary to promote the cooperation between HVN stations and nursing schools to increase practical HVN training for pre-graduation student nurses.

\section{Acknowledgements}

The authors thank all the respondents who completed the questionnaire. There was no incentive or reward offered in this respect, so we appreciate they freely donated their valuable time and shared their views to answer and comment in this study. We had many useful comments in the open-response final-section in our questionnaire. Many of these comments will help inform future research. This research was funded by a Grant-in-Aid for Scientific Research C (grant number 18K10538) from the Japanese Ministry of Education, Culture, Sports, Science and Technology. 


\section{References}

1. Nishino T. Quantitative properties of the macro supply and demand structure for care facilities for elderly in Japan. Int J Environ Res Public Health 2017; 14: 1489-1511. [Medline] [CrossRef]

2. Ministry of Economy, Trade and Industry. Study Group for Future Supply and Demand of Elderly Nursing Care Systems. https://www.meti.go.jp/pre ss/2018/04/20180409004/20180409004-2.pdf (Accessed August 13, 2021) (in Japanese)

3. Ministry of Health, Labour and Welfare. Nurse Supply and Demand Subcommittee Report. https://www.mhlw.go.jp/stf/shingi/other-isei_338805.html (Accessed August 13, 2021) (in Japanese)

4. Japan Visiting Nursing Foundation. Houmon-Kango Action Plan 2025. Houmonkango-Suisin-Renkei-Kaigi. https://www.jvnf.or.jp/2017/actionplan2025. pdf (Accessed April 27, 2021) (in Japanese)

5. Japanese Nursing Association. Surveys on Home-Visiting Nurse 2014. https://www.nurse.or.jp/home/publication/pdf/report/2015/homonjittai.pdf (Accessed August 13, 2021) (in Japanese)

6. van Iersel M, Latour CH, de Vos R, et al. Nursing students' perceptions of community care and other areas of nursing practice - A review of the literature. Int J Nurs Stud 2016; 61: 1-19. [Medline] [CrossRef]

7. Abiodun RO, Daniels F, Pimmer C, et al. Nurse graduates' experiences and support needs: a qualitative systematic review of South Africa's community service programme. Curationis 2019; 42: e1-e12. [Medline] [CrossRef]

8. Akiyama A, Fukuyama Y. Qualities required for new graduate visiting nurses: a qualitative study. J Gen Fam Med 2020; 21: 87-91. [Medline] [CrossRef]

9. Fukuyama Y, Akiyama A. Home-care nursing challenges for newly qualified nurses: a qualitative study. Int J Gerontol 2019, 78-82.

10. The National Association for Visiting Nurse Service. Government Health and Medical Service Grants for the Elderly 2018. Research project on the education system for home-visiting nurse station to hire and train new graduates nurses. https://www.zenhokan.or.jp/wp-content/uploads/h29-2.pdf (Accessed April 27, 2021) (in Japanese)

11. Maruyama Y, Kashiwagi M, Kanoya Y. The present situation and related factors of employment of and intention to employ novice nurses by home-visit nursing stations. J Jpn Health Med Assoc 2018; 27: 347-353 (in Japanese).

12. Narahara R, Nishiyama Y. Workplace environment to support new graduate visiting nurse. Care. Nursing Journal of SHIJONAWATE GAKUEN UNIVERSITY. 2017; 1: 37-44 (in Japanese).

13. Chen FQ, Leng YF, Ge JF, et al. Effectiveness of virtual reality in nursing education: meta-analysis. J Med Internet Res 2020; 22: e18290. [Medline] [CrossRef]

14. Connell CJ, Endacott R, Jackman JA, et al. The effectiveness of education in the recognition and management of deteriorating patients: a systematic review. Nurse Educ Today 2016; 44: 133-145. [Medline] [CrossRef] 